\title{
Univariate and multivariate analyses of preoperative factors influencing symptomatic outcomes of transoral fundoplication
}

\author{
Reginald C. W. Bell • Mark A. Fox • William E. Barnes • Peter G. Mavrelis • \\ Robert W. Sewell • Bart J. Carter • Glenn M. Ihde • Karim S. Trad • \\ David Dargis • Kevin M. Hoddinott • Katherine D. Freeman • Tanja Gunsberger • \\ Mark G. Hausmann • Brian DaCosta Gill • Erik Wilson
}

Received: 10 January 2014/ Accepted: 12 April 2014/Published online: 31 May 2014

(C) The Author(s) 2014. This article is published with open access at Springerlink.com

\begin{abstract}
Background Preoperative factors predicting symptomatic improvement after transoral fundoplication (TF) in chronic gastroesophageal reflux disease (GERD) patients with persistent symptoms on proton-pump inhibitors (PPIs) therapy have not been elucidated fully.

Methods Univariate and multivariate logistic regression analyses were performed on data from 158 consecutive patients who underwent TF with the EsophyX device between January 2010 and June 2012 in 14 community centers. Variables included age, gender, body mass index,
\end{abstract}

This work was presented as a poster at the 2014 meeting of the Society of American Gastrointestinal and Endoscopic Surgeons (SAGES), Salt Lake City, UT.

R. C. W. Bell $(\bowtie) \cdot$ K. D. Freeman

SurgOne Foregut Institute, 401 West Hampden Place, Suite 230, Englewood, CO 80110, USA

e-mail: rbell@surgone.com

M. A. Fox

Crossville Medical Group, PA, Crossville, TN, USA

W. E. Barnes

Livingston Hospital and Healthcare Services, Inc. CAH, Salem, KY, USA

P. G. Mavrelis

Internal Medicine Associates, Merrillville, IN, USA

R. W. Sewell

Master Center for Minimally Invasive Surgery, Southlake, TX, USA

B. J. Carter

Mt. Graham Regional Medical Center, Safford, AZ, USA

G. M. Ihde

Ihde Surgical Group, Arlington, TX, USA
GERD duration, PPIs therapy duration, presence of hiatal hernia, esophagitis, Hill grade, quality of life scores (QOL) on PPIs, \% total time $\mathrm{pH}<4$, and DeMeester score on reflux testing off PPIs.

Results All patients suffered from typical GERD symptoms. Additionally, $78 \%(124 / 158)$ of patients suffered from atypical symptoms. Six percent (10/158) with recurrent GERD symptoms refractory to PPI therapy underwent revisional procedure (9 laparoscopic Nissen, 1 TF). Median follow-up was 22 (range 10-43) months. For patients with typical symptoms, univariate analyses revealed 4 preoperative factors predictive of successful outcomes: age $\geq 50$ [odds ratio $(\mathrm{OR})=2.4,95 \%$ confidence interval $(C I)=1.2-4.8, p=0.014]$, GERD Health-related Quality of Life score (GERD-HRQL) $\geq 15$ on PPIs $(\mathrm{OR}=6.0$,

\section{K. S. Trad}

The George Washington University School of Medicine and Health Sciences, Washington, DC, USA

\section{K. S. Trad}

Reston Surgical Associates, Reston, VA, USA

D. Dargis

Allegan Surgical Associates, Allegan, MI, USA

K. M. Hoddinott

Munroe Regional Medical Center, Ocala, FL, USA

T. Gunsberger

Tempe St. Luke's Hospital, Tempe, AZ, USA

M. G. Hausmann

The Surgeons Group of Baton Rouge, Baton Rouge, LA, USA

B. D. Gill

Utah County Surgical Associates, Provo, UT, USA

E. Wilson

University of Texas Health Science Center, Houston, TX, USA 
$\mathrm{CI}=1.2-29.4, p=0.026$, Reflux Symptom Index score $>13$ on PPIs $(\mathrm{OR}=2.4, \mathrm{CI}=1.1-5.2, p=0.027)$, and Gastroesophageal Reflux Symptom Score $\geq 18$ on PPIs $(\mathrm{OR}=2.6, \mathrm{CI}=1.2-5.8, p=0.018)$. Age and GERDHRQL score remained significant predictors by multivariate analysis. For patients with atypical symptoms, only GERD-HRQL $\quad$ score $\geq 15$ on PPIs $\quad(\mathrm{OR}=9.9$, $\mathrm{CI}=0.9-4.6, p=0.036)$ was associated with successful outcomes.

Conclusions Elevated preoperative QOL scores on PPIs and age $\geq 50$ were most closely associated with successful outcome of TF in patients with persistent symptoms despite medical therapy.

Keywords Heartburn - EsophyX - Gastroesophageal reflux · TIF · Regurgitation - GERD · Refractory

The successful management of chronic gastroesophageal reflux disease (GERD) includes controlling troublesome reflux symptoms, improving patients' quality of life (QOL), and preventing complications [1, 2]. Despite the prevalent use of lifestyle modification, high-dose protonpump inhibitor (PPI) therapy and other medications, 20-40\% of medically treated GERD patients continue to have persistent troublesome symptoms [3]. Although treatment of GERD with laparoscopic fundoplication (LF), when performed in specialty centers, historically reports an excellent relief of typical GERD symptoms [4], the procedure is performed in a minor percentage of patients with persistent symptoms despite medical therapy and nationally this number is decreasing. Presence of typical symptoms and good response to PPI therapy have predicted success with LF treatment [5]. Partly with the goal of providing better outcomes to these patients with persistent symptoms despite medical therapy, endoscopic antireflux procedures have been evaluated as potential alternatives to those who do not wish to have LF. This study evaluated factors that might be predictive of successful symptomatic results after one such endoscopic antireflux procedure.

Transoral fundoplication (TF) using the EsophyX device has been described previously [6-10]. Under general anesthesia, the device is introduced over a flexible endoscope into the esophagus and stomach. A tissue mold at the end of the device apposes the gastric fundus to the distal esophagus, and small $(6-7 \mathrm{~mm})$ polypropylene H-shaped fasteners are placed from esophageal to fundic lumen to create the plication. An endoluminal esophagogastric fundoplication can be created up to $4 \mathrm{~cm}$ in length and $270^{\circ}$ circumference as confirmed by endoscopic evaluation.

Although some reports of TF evaluated variables associated with clinical outcomes as a portion of the report $[6$,
11-13], to date there is no single study that primarily investigated association of preoperative factors with symptomatic outcomes of TF. In this study, we evaluated multiple preoperative factors and their relationship to clinical outcomes of TF in GERD patients with an inadequate response to PPIs.

\section{Patients and methods}

Study design

A prospective 14-center community practice registry was established in 2010 (Clinicaltrials.gov identifier NCT01118585). The primary endpoint of the study was elimination of troublesome GERD symptoms in patients undergoing TF using the EsophyX device. None of the patients in the study had concomitant crural closure. Outcomes were evaluated using disease-specific questionnaires. The current study evaluated patient variables with regard to the success of the procedure.

The following preoperative measures were recorded: age, gender, body mass index (BMI), GERD duration, PPI therapy duration, presence of hiatal hernia, Los Angeles (LA) classification of esophagitis, Hill grade of the gastroesophageal (GE) valve, and GERD-specific QOL scores on PPIs. The QOL questionnaires administered were the GERD Health-related Quality of Life (GERD-HRQL) score, Reflux Symptom Index (RSI) score, and Gastroesophageal Reflux Symptom Score (GERSS).

\section{Study variables}

Variables were divided into binary groups for analysis as follow: gender (male or female), age $(<50$ or $\geq 50$ years old), GERD duration ( $<5$ or $\geq 5$ years), duration of PPI use ( $<5$ or $\geq 5$ years), BMI $(<30$ or $\geq 30$ ), hiatal hernia (present or absent), esophagitis (present or absent), GERD$\mathrm{HRQL}<15$ or $\geq 15, \quad \mathrm{RSI} \leq 13$ or $>13, \quad$ GERSS $<18$ or $\geq 18$. Use of proton-pump inhibitors were categorized as daily, occasional, or none.

Outcomes were assessed using QOL questionnaires and recording of acid-suppressive medication use at defined postoperative time periods $(6,12,24$, and 36 months).

\section{Patient population}

The study population consisted of 158 patients enrolled in the multicenter registry who underwent TF with EsophyX device without crural closure. The procedures were performed in 14 community-based centers across the United 
States between January 2010 and June 2012 following the previously described 2.0 protocol [14]. Primary inclusion criteria were (1) GERD symptoms for at least 1 year, (2) history of daily PPI use for at least 6 months even if patients were not currently taking PPIs, (3) hiatal hernia $\leq 2 \mathrm{~cm}$ in axial length and $\leq 3 \mathrm{~cm}$ in greatest transverse dimension, and (4) willingness to provide informed consent. Primary exclusion criteria were (1) a BMI greater than $35 \mathrm{~kg} / \mathrm{m}^{2}$, (2) esophagitis grade D (LA classification), (3) GE junction classified as Hill grade IV, (4) advanced disease including long segment Barrett's esophagus, esophageal ulcer, and fixed esophageal stricture or narrowing. All patients in this study had typical GERD symptoms and objective documentation of GERD.

Preoperative evaluation

Preoperative evaluation included symptom assessment using GERD-HRQL, RSI, and GERSS questionnaires. Objective documentation of GERD was obtained from upper endoscopy findings of esophagitis, non-fixed stricture, columnar lined epithelium with Barrett esophagus on biopsy (limited to $\leq 2 \mathrm{~cm}$ by study protocol), and/or abnormal ambulatory reflux testing.

Intervention and postoperative care

Transoral esophagogastric endoscopic fundoplication using the EsophyX $\mathrm{X}_{2}$ device (EndoGastric Solutions, Inc, Redmond, Washington) without crural repair was performed using a defined protocol (2.0). The main goal of the procedure was to create a GE valve $270^{\circ}$ or more in circumference and $2-5 \mathrm{~cm}$ in length. This was achieved by endoluminal folding of the fundus of the stomach around the distal esophagus and securing it in place with multiple (12-30) " $\mathrm{H}$ "-shaped polypropylene fasteners. Detailed technical aspects of the procedure have been previously described [14].

Following the procedure, patients were asked to continue PPI therapy for 2 weeks and to adhere to a modified diet for 4-6 weeks. Anti-emetic prophylaxis was routinely administered in the first $24 \mathrm{~h}$ after the procedure and then as needed. Patients were asked to desist from rigorous physical activities for 4-6 weeks after the procedure.

\section{Outcome measures}

Three validated self-reported disease-specific questionnaires evaluated symptom severity prior to and after the procedure. The GERD-HRQL evaluates heartburn (six questions), dysphagia (two), bloating (one), and the impact of medication on daily life (one) on a scale from 0 (no symptoms) to 5 (incapacitating symptoms). The scale combines frequency and severity of symptoms in a single response. Total score on the GERD-HRQL ranges from 0 to 50, with higher scores indicating more severe GERD $[15,16]$. There is not a defined "normal" score; instead a $50 \%$ improvement in total GERD-HRQL score is indicative of a successful therapeutic outcome. RSI measures symptoms associated with extraesophageal manifestations of GERD (atypical symptoms) [17]. Each of nine symptom scores can range from 0 (no symptoms) to 5 (severe symptoms), with a maximum total score of 45 . A total RSI score of $\leq 13$ is considered normal [17]. The GERSS questionnaire assesses classic (heartburn and regurgitation) and atypical GERD symptoms (abdominal distension, dysphagia, and cough) [18, 19]. Specific symptom items are scored as a product of severity $(0=$ no symptoms at all to $3=$ severe symptoms $)$ and frequency $(0=$ never to $4=$ daily). These five symptom scores (range 0-12) are then summed to create the total GERSS score (range 0-60). A total GERSS $<18$ indicates controlled reflux symptoms and is considered normal [19]. All patients completed preoperative questionnaires on PPIs. At follow-up, patients completed QOL questionnaires without altering their current GERD medical treatment, if any.

PPI usage was defined as "none" (medication not taken at all), "occasional" (if any dose was taken $\leq 3$ days a week), or "daily" (if any dose was taken $>3$ days a week).

Definition of symptomatic treatment outcomes: successful, responsive, poor

Clinical outcome was considered successful if patients experienced $\geq 50 \%$ reduction of a GERD-HRQL score or normalization of RSI score $(\leq 13)$ and were completely off PPIs. Outcome was considered responsive if patients experienced $\geq 50 \%$ reduction in GERD-HRQL score or normalization of RSI regardless of ongoing medication use (as long as the dosing of medication did not increase). An outcome was considered poor for patients who experienced a $<50 \%$ reduction in GERD-HRQL or had abnormal RSI at follow-up, or underwent reoperation or increased their medication use after surgery. Analyses were performed separately on patients with typical and atypical symptoms before TF.

Data collection and statistical analyses

All data were prospectively collected. Descriptive statistics were calculated for variables of interest. Univariate and multivariate logistic regression analyses were performed to identify factors predictive of successful symptomatic outcomes following TF. Covariate with Wald's $p$ values $\leq 0.25$ on univariate analyses was entered into backwards stepwise multivariate regression analyses. 


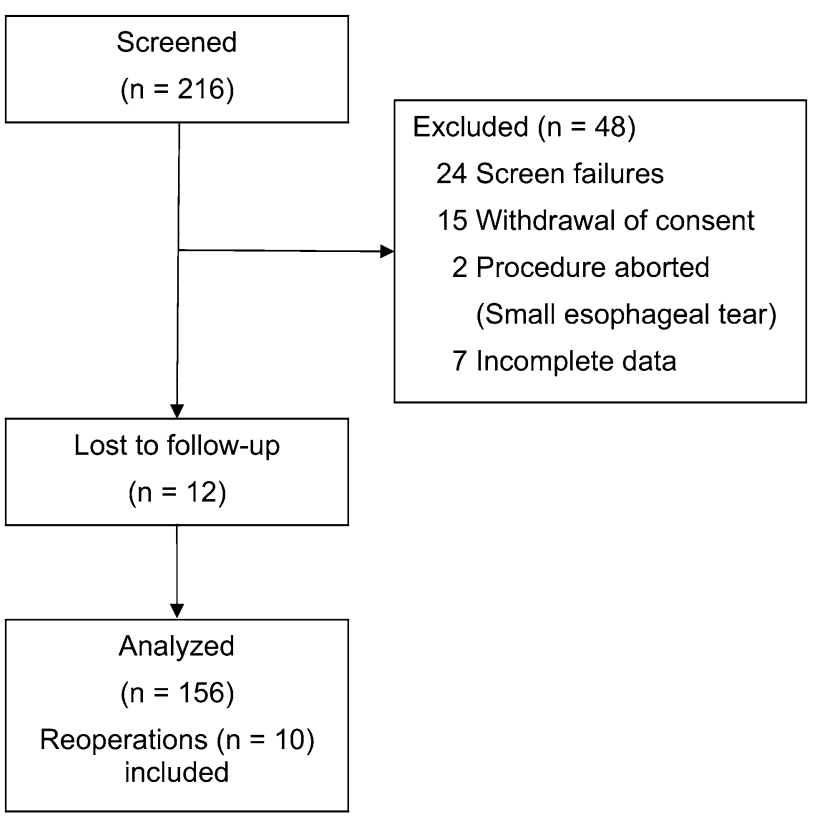

Fig. 1 Flowchart of patients enrolled in the study

$p$ value $\leq 0.05$ was considered significant. Descriptive results are reported as median (range). Categorical data were reported as proportions and counts. Individual nonparametric Wilcoxon signed rank tests were performed to compare improvement in QOL scores at 22-month followup versus baseline. McNemar's test was performed to compare proportions of paired data. A $p$ value $<0.05$ was considered to be statistically significant for these tests. All analyses were performed using JMP 10.0 statistical program.

\section{Results}

\section{Patient characteristics}

This study enrolled 158 patients with medically refractory typical GERD symptoms who underwent TF without crural repair between January 2010 and June 2012 (Fig. 1). Median follow-up was 22 (range 10-43) months. The median age was 59 years (range 19-90 years), and $29 \%$ of patients were male. The study population included 10 patients ( $6 \%$ of study population) who developed recurrent GERD symptoms after TF and decided to undergo a revisional procedure (9 laparoscopic Nissen fundoplication and $1 \mathrm{TF})$. For QOL comparisons, these patients were considered to have poor outcomes, and preoperative QOL scores were imputed for follow-up visits. Demographics and baseline characteristics of study patients are summarized in Table 1.
Table 1 Baseline characteristics of study patients

\begin{tabular}{ll}
\hline Characteristics & Frequency \\
$(n=158)$
\end{tabular}

Gender

Male, $n(\%)$

Female, $n(\%)$

Age, median (range)

$58.5(19-90)$

$<50, n(\%)$

$\geq 50, n(\%)$

$111(70)$

Body mass index, $\mathrm{kg} / \mathrm{m}^{2}$

$<30, n(\%)$

$117(74)$

$\geq 30, n(\%)$

41 (26)

Esophagitis (Los Angeles Grade), $n(\%)$

$107(68)$

$\mathrm{A}, n(\%)$

$30(28)$

$\mathrm{B}, n(\%)$

$63(59)$

$\mathrm{C}, n(\%)$

Hiatal hernia

Yes, $n(\%)$

$113(72)$

No, $n(\%)$

GERD duration $^{\mathrm{a}}$

$<5, n(\%)$

$\geq 5, n(\%)$

$110(77)$

PPI duration ${ }^{\mathrm{a}}$

$<5, n(\%)$

$\geq 5, n(\%)$

GERD-HRQL score on PPI therapy

$<15, n(\%)$

$\geq 15, n(\%)$

RSI score on PPI therapy ${ }^{\mathrm{b}}$

$\leq 13, n(\%)$

$>13, n(\%)$

GERSS score on PPI therapy ${ }^{\mathrm{c}}$

$<18, n(\%)$

$\geq 18, n(\%)$
$\%$ total time $\mathrm{pH}<4^{\mathrm{d}}$

$\geq 8 \%, n / n(\%)$

$13 / 27(48)$

$<8 \%, n / n(\%)$

$14 / 27(52)$

Total DeMeester score on 48 -h testing ${ }^{\mathrm{e}}$

$\geq 30, n / n(\%)$

$<30, n / n(\%)$

$16 / 23(70)$

$G E R D$ gastroesophageal reflux disease, GERD-HRQL gastroesophageal reflux disease health-related quality of life, $R S I$ reflux symptom index, GERSS gastroesophageal reflux symptom score, PPI protonpump inhibitor

${ }^{a}$ Data were available for 144 patients

b One patient with incomplete questionnaire was excluded form analyses

c Six patients with incomplete questionnaires were excluded from analyses

${ }^{\mathrm{d}}$ Data were available for 27 patients

${ }^{\text {e }}$ Data were available for 23 patients 
Table 2 Univariate regression analyses for successful resolution of classic GERD symptoms

\begin{tabular}{|c|c|c|c|}
\hline Variable & $\begin{array}{l}\text { Successful } \\
\text { outcome }\end{array}$ & $\begin{array}{l}\text { Unadjusted OR } \\
(95 \% \mathrm{CI})\end{array}$ & $p$ value \\
\hline \multicolumn{4}{|c|}{ Age (years) } \\
\hline$<50$ & $20 / 47(43)$ & & \\
\hline$\geq 50$ & $71 / 111(64)$ & $2.4(1.2-4.9)$ & 0.014 \\
\hline \multicolumn{4}{|l|}{ Gender } \\
\hline Male & $21 / 46(46)$ & & \\
\hline Female & $70 / 112(63)$ & $2.0(1.0-4.0)$ & 0.053 \\
\hline \multicolumn{4}{|c|}{ Body mass index $\left(\mathrm{kg} / \mathrm{m}^{2}\right)$} \\
\hline$<30$ & $67 / 117(57)$ & & \\
\hline$\geq 30$ & $24 / 41(59)$ & $1.1(0.5-2.2)$ & 0.887 \\
\hline \multicolumn{4}{|c|}{ Hiatal hernia } \\
\hline No & $22 / 45(49)$ & & \\
\hline Yes & $69 / 113(61)$ & $1.6(0.8-3.3)$ & 0.164 \\
\hline \multicolumn{4}{|c|}{ Esophagitis } \\
\hline No & $24 / 51(47)$ & & \\
\hline Yes & $67 / 107(63)$ & $1.9(1.0-3.7)$ & 0.065 \\
\hline \multicolumn{4}{|c|}{ GERD duration (years) } \\
\hline$<5$ & $20 / 34(59)$ & & \\
\hline$\geq 5$ & $63 / 112(56)$ & $0.9(0.4-2.0)$ & 0.862 \\
\hline \multicolumn{4}{|c|}{ PPI duration (years) } \\
\hline$<5$ & $31 / 52(60)$ & & \\
\hline$\geq 5$ & $51 / 92(55)$ & $0.8(0.4-1.7)$ & 0.627 \\
\hline \multicolumn{4}{|c|}{ GERD-HRQL score on PPIs } \\
\hline$<15$ & $2 / 10(20)$ & & \\
\hline$\geq 15$ & $89 / 148(60)$ & $6.0(1.5-40.1)$ & 0.026 \\
\hline \multicolumn{4}{|c|}{ RSI score on PPIs } \\
\hline$\leq 13$ & $14 / 34(41)$ & & \\
\hline$>13$ & $77 / 123(63)$ & $2.4(1.1-5.3)$ & 0.027 \\
\hline \multicolumn{4}{|c|}{ GERSS score on PPIs } \\
\hline$<18$ & $13 / 32(41)$ & & \\
\hline$\geq 18$ & $77 / 120(64)$ & $2.6(1.2-5.9)$ & 0.018 \\
\hline \multicolumn{4}{|c|}{$\%$ total time $\mathrm{pH}<4$} \\
\hline$\geq 8 \%$ & $4 / 13(31)$ & & \\
\hline$<8 \%$ & $7 / 14(50)$ & $2.3(0.5-11.7)$ & 0.313 \\
\hline \multicolumn{4}{|c|}{ Total DeMeester score } \\
\hline$\geq 30$ & 2/7 (29) & & \\
\hline$<30$ & $8 / 16(50)$ & $1.8(0.3-10.4)$ & 0.539 \\
\hline
\end{tabular}

Data are expressed n/n (\%)

$G E R D$ gastroesophageal reflux disease, GERD-HRQL gastroesophageal reflux disease health-related quality of life, GERSS gastroesophageal reflux symptom score, $O R$ odds ratio, $P P I s$ proton-pump inhibitors, RSI reflux symptom index

${ }^{\text {a }}$ Wald $p$ value

In addition to having typical GERD symptoms, $78 \%$ $(124 / 158)$ of patients suffered from atypical symptoms (RSI score $>13$ ). An additional analysis was conducted looking for predictive factors for outcomes of atypical symptoms in these patients.
Table 3 Multivariate backward stepwise regression analysis for successful resolution of classic GERD symptoms

\begin{tabular}{|c|c|c|c|c|}
\hline \multirow[t]{2}{*}{ Variable } & \multicolumn{2}{|c|}{ Successful outcome } & \multicolumn{2}{|c|}{$\begin{array}{l}\text { Successful or responsive } \\
\text { outcome }\end{array}$} \\
\hline & OR $(95 \% \mathrm{CI})$ & $p$ value & OR $(95 \% \mathrm{CI})$ & $p$ value \\
\hline \multicolumn{5}{|c|}{ Age (years) } \\
\hline$<50$ & - & - & - & - \\
\hline$\geq 50$ & $2.6(1.3-5.3)$ & 0.008 & $2.6(1.2-5.7)$ & 0.018 \\
\hline \multicolumn{5}{|c|}{ GERD-HRQL score on PPIs } \\
\hline$<15$ & - & - & - & - \\
\hline$\geq 15$ & $7.1(1.7-48.5)$ & 0.017 & - & - \\
\hline \multicolumn{5}{|c|}{ Esophagitis } \\
\hline No & - & - & - & - \\
\hline Yes & - & - & $2.9(1.3-6.3)$ & 0.008 \\
\hline
\end{tabular}

GERD-HRQL gastroesophageal reflux disease health-related quality of life, $O R$ odds ratio, PPIs proton-pump inhibitors

Patients with typical GERD symptoms

All 158 patients had preoperative troublesome typical GERD symptoms on PPIs, defined as having at least one of the individual items on GERD-HRQL scored $>2$. Additionally, all 158 patients had a total GERD-HRQL $\geq 10$ on PPIs at entry. The median GERD-HRQL score improved from 28 $(10-50)$ on PPIs to $5(0-45)$ at follow-up, $p<0.001 .75 \%$ of patients $(118 / 158)$ experienced a $\geq 50 \%$ reduction in their GERD-HRQL score. All patients were on PPIs at screening. Complete cessation of PPI therapy occurred in $70 \%$ (110/ 158) of patients; an additional $7 \%$ (11/158) of patients were only taking PPIs occasionally. $23 \%(37 / 158)$ of patients remained on daily PPI therapy. No patient had increased their PPI dose at follow-up as compared with baseline. A responsive outcome was achieved in $75 \%$ (119/158) of patients $(\geq 50 \%$ reduction of GERD-HRQL regardless of continuing medication use). A successful outcome was achieved in $58 \%$ (91/158) of patients ( $\geq 50 \%$ reduction of GERD-HRQL and no PPI use). Outcomes were considered poor in $25 \%$ (39/158) of patients with typical symptoms.

Predictive factors of a successful outcome (off PPIs and $\geq 50 \%$ improvement in the total GERD-HRQL score) by univariate analysis were (1) age $\geq 50$ [odds ratio $(\mathrm{OR})=2.4,95 \%$ confidence interval $(\mathrm{CI})=1.2-4.9$, $p=0.014]$, (2) preoperative GERD-HRQL score $\geq 15$ on PPIs (OR $=6.0, \mathrm{CI}=1.5-40.1, p=0.026)$, (3) preoperative RSI score $>13$ on PPIs $(\mathrm{OR}=2.4, \mathrm{CI}=1.1-5.3$, $p=0.027$ ), and (4) preoperative GERSS score $\geq 18$ on PPIs (OR $=2.6, \mathrm{CI}=1.2-5.9, p=0.018)$. Female gender $(\mathrm{OR}=2.0, \mathrm{CI}=1.0-4.0, p=0.053)$ and presence of esophagitis on endoscopy $(\mathrm{OR}=1.9, \quad \mathrm{CI}=1.0-3.7$, $p=0.065$ ) closely approached significant level (Table 2). By multivariate analysis, age $\geq 50 \quad(\mathrm{OR}=2.6, \mathrm{CI}=$ $1.3-5.3, p=0.008$ ) and initial GERD-HRQL score $\geq 15$ on 
Table 4 Univariate regression analyses for successful or responsive resolution of classic GERD symptoms

\begin{tabular}{|c|c|c|c|}
\hline Variable & $\begin{array}{l}\text { Successful or responsive } \\
\text { outcome }\end{array}$ & $\begin{array}{l}\text { Unadjusted OR } \\
(95 \% \mathrm{CI})\end{array}$ & $p$ value $\mathrm{e}^{\mathrm{a}}$ \\
\hline \multicolumn{4}{|c|}{ Age (years) } \\
\hline$<50$ & $30 / 47(64)$ & & \\
\hline$\geq 50$ & $89 / 111(80)$ & $2.3(1.1-4.9)$ & 0.032 \\
\hline \multicolumn{4}{|l|}{ Gender } \\
\hline Male & $30 / 46(65)$ & & \\
\hline Female & 89/112 (80) & $2.1(1.0-4.4)$ & 0.062 \\
\hline \multicolumn{4}{|c|}{ Body mass index $\left(\mathrm{kg} / \mathrm{m}^{2}\right)$} \\
\hline$<30$ & $88 / 117(75)$ & & \\
\hline$\geq 30$ & $31 / 41(76)$ & $1.0(0.5-2.4)$ & 0.960 \\
\hline \multicolumn{4}{|c|}{ Hiatal hernia } \\
\hline No & $31 / 45(69)$ & & \\
\hline Yes & $88 / 113(78)$ & $1.6(0.7-3.4)$ & 0.239 \\
\hline \multicolumn{4}{|c|}{ Esophagitis } \\
\hline No & $32 / 51(63)$ & & \\
\hline Yes & $87 / 107(81)$ & $2.6(1.2-5.5)$ & 0.013 \\
\hline \multicolumn{4}{|c|}{ GERD duration } \\
\hline$<5$ & 27/34 (79) & & \\
\hline$\geq 5$ & $84 / 112(75)$ & $0.8(0.3-1.9)$ & 0.598 \\
\hline \multicolumn{4}{|c|}{ PPI duration } \\
\hline$<5$ & $38 / 52(73)$ & & \\
\hline$\geq 5$ & 71/92 (77) & $1.2(0.6-2.7)$ & 0.582 \\
\hline \multicolumn{4}{|c|}{ GERD-HRQL score on PPIs } \\
\hline$<15$ & $5 / 10(50)$ & & \\
\hline$\geq 15$ & $114 / 148(77)$ & $3.4(0.9-12.7)$ & 0.068 \\
\hline \multicolumn{4}{|c|}{ RSI score on PPIs } \\
\hline$\leq 13$ & $22 / 34(65)$ & & \\
\hline$>13$ & $96 / 123(78)$ & $1.9(0.8-4.4)$ & 0.115 \\
\hline \multicolumn{4}{|c|}{ GERSS score on PPIs } \\
\hline$<18$ & $20 / 32(63)$ & & \\
\hline$\geq 18$ & $96 / 120(80)$ & $2.4(1.0-5.6)$ & 0.042 \\
\hline \multicolumn{4}{|c|}{$\%$ total time $\mathrm{pH}<4$} \\
\hline$\geq 8 \%$ & $6 / 13(46)$ & & \\
\hline$<8 \%$ & $8 / 14(57)$ & $1.6(0.3-7.4)$ & 0.569 \\
\hline \multicolumn{4}{|c|}{ Total DeMeester score } \\
\hline$\geq 30$ & $3 / 7(43)$ & & \\
\hline$<30$ & $9 / 16(56)$ & $1.3(0.3-6.4)$ & 0.736 \\
\hline
\end{tabular}

Data are expressed n/n (\%)

$G E R D$ gastroesophageal reflux disease, GERD-HRQL gastroesophageal reflux disease health-related quality of life, GERSS gastroesophageal reflux symptom score, $O R$ odds ratio, $P P I s$ proton-pump inhibitors, $R S I$ reflux symptom index

${ }^{\text {a }}$ Wald $p$ value

PPIs $(\mathrm{OR}=7.1, \mathrm{CI}=1.7-47.5, p=0.017)$ were predictive of a successful outcome (Table 3). When age was $\geq 50$ and GERD-HRQL score was $\geq 15$ on PPIs preoperatively, a successful outcome was achieved in $67 \%$ (68/102) of patients
Table 5 Univariate regression analyses for successful resolution of atypical GERD symptoms

\begin{tabular}{|c|c|c|c|}
\hline Variable & $\begin{array}{l}\text { Successful } \\
\text { outcome }\end{array}$ & $\begin{array}{l}\text { Unadjusted OR } \\
(95 \% \text { CI })\end{array}$ & $p$ value $^{\mathrm{a}}$ \\
\hline \multicolumn{4}{|c|}{ Age (years) } \\
\hline$<50$ & $20 / 36(56)$ & & \\
\hline$\geq 50$ & $54 / 88(61)$ & $1.3(0.6-2.8)$ & 0.550 \\
\hline \multicolumn{4}{|l|}{ Gender } \\
\hline Male & $15 / 30(50)$ & & \\
\hline Female & $59 / 94(63)$ & $1.7(0.7-3.9)$ & 0.217 \\
\hline \multicolumn{4}{|c|}{ Body mass index $\left(\mathrm{kg} / \mathrm{m}^{2}\right)$} \\
\hline$<30$ & $52 / 92(57)$ & & \\
\hline$\geq 30$ & $22 / 32(59)$ & $1.7(0.7-4.1)$ & 0.227 \\
\hline \multicolumn{4}{|c|}{ Hiatal hernia } \\
\hline No & $17 / 35(49)$ & & \\
\hline Yes & $57 / 89(64)$ & $1.9(0.9-4.2)$ & 0.116 \\
\hline \multicolumn{4}{|c|}{ Esophagitis } \\
\hline No & $15 / 32(47)$ & & \\
\hline Yes & $59 / 92(64)$ & $2.0(0.9-4.6)$ & 0.089 \\
\hline \multicolumn{4}{|c|}{ GERD duration } \\
\hline$<5$ & $17 / 27(63)$ & & \\
\hline$\geq 5$ & $52 / 87(604)$ & $0.9(0.3-2.1)$ & 0.767 \\
\hline \multicolumn{4}{|c|}{ PPI duration } \\
\hline$<5$ & $28 / 42(67)$ & & \\
\hline$\geq 5$ & $41 / 71(58)$ & $0.7(0.3-1.5)$ & 0.348 \\
\hline
\end{tabular}

GERD-HRQL score on PPIs

$\begin{array}{lrrr}<15 & 1 / 7(14) & & \\ \geq 15 & 73 / 117(62) & 10.0(1.6-191.2) & 0.036\end{array}$

RSI score on PPIs

$\begin{array}{lr}\leq 13 & 0 / 0(0) \\ >13 & 74 / 124(60)\end{array}$

GERSS score on PPIs

$\begin{array}{lrrr}<18 & 7 / 12(58) & & \\ \geq 18 & 67 / 110(61) & 1.1(0.3-3.7) & 0.862 \\ \% \text { total time } \mathrm{pH}<4 & & \\ \geq 8 \% & 4 / 11(36) & & \\ <8 \% & 3 / 8(38) & 1.1(0.1-7.1) & 0.960\end{array}$

Total DeMeester score

$\begin{array}{lrrr}\geq 30 & 1 / 5(20) & & \\ <30 & 4 / 11(36) & 2.3(0.2-53.4) & 0.519\end{array}$

Data are expressed n/n (\%)

$G E R D$ gastroesophageal reflux disease, GERD-HRQL gastroesophageal reflux disease health-related quality of life, GERSS gastroesophageal reflux symptom score, $O R$ odds ratio, PPIs proton-pump inhibitors, $R S I$ reflux symptom index

${ }^{\text {a }}$ Wald $p$ value

with inadequate control of symptoms despite medical therapy. Inversely, if both factors were unfavorable (age $<50$ and preoperative GERD-HRQL score on PPIs < 15), $20 \%$ (3/15) of patients achieved a successful outcome. 
Table 6 Univariate regression analyses for successful or responsive resolution of atypical GERD symptoms

\begin{tabular}{|c|c|c|c|}
\hline Variable & $\begin{array}{l}\text { Successful or } \\
\text { responsive } \\
\text { outcome }\end{array}$ & $\begin{array}{l}\text { Unadjusted OR } \\
(95 \% \mathrm{CI})\end{array}$ & $p$ value $^{\mathrm{a}}$ \\
\hline \multicolumn{4}{|c|}{ Age (years) } \\
\hline$<50$ & $26 / 36(72)$ & & \\
\hline$\geq 50$ & $67 / 88(76)$ & $1.2(0.5-2.9)$ & 0.648 \\
\hline \multicolumn{4}{|l|}{ Gender } \\
\hline Male & $20 / 30(67)$ & & \\
\hline Female & 73/94 (78) & $1.7(0.7-4.2)$ & 0.229 \\
\hline \multicolumn{4}{|c|}{ Body mass index $\left(\mathrm{kg} / \mathrm{m}^{2}\right)$} \\
\hline$<30$ & $67 / 92(73)$ & & \\
\hline$\geq 30$ & $26 / 32(81)$ & $1.6(0.6-4.8)$ & 0.346 \\
\hline \multicolumn{4}{|c|}{ Hiatal hernia } \\
\hline No & $22 / 35(63)$ & & \\
\hline Yes & $71 / 89(80)$ & $2.3(1.0-5.5)$ & 0.535 \\
\hline \multicolumn{4}{|c|}{ Esophagitis } \\
\hline No & $18 / 32(56)$ & & \\
\hline Yes & $75 / 92(82)$ & $3.4(1.4-8.3)$ & 0.006 \\
\hline \multicolumn{4}{|c|}{ GERD duration } \\
\hline$<5$ & $17 / 27(63)$ & & \\
\hline$\geq 5$ & $52 / 87(60)$ & $0.9(0.3-2.1)$ & 0.767 \\
\hline \multicolumn{4}{|c|}{ PPI duration } \\
\hline$<5$ & $28 / 42(67)$ & & \\
\hline$\geq 5$ & $41 / 71(58)$ & $0.7(0.3-1.5)$ & 0.348 \\
\hline \multicolumn{4}{|c|}{ GERD-HRQL score on PPIs } \\
\hline$<15$ & 4/7 (57) & & \\
\hline$\geq 15$ & $89 / 117(76)$ & $2.4(0.4-11.4)$ & 0.274 \\
\hline \multicolumn{4}{|c|}{ RSI score on PPIs } \\
\hline$\leq 13$ & $0 / 0(0)$ & & \\
\hline$>13$ & $93 / 124(75)$ & - & - \\
\hline \multicolumn{4}{|c|}{ GERSS score on PPIs } \\
\hline$<18$ & $8 / 12(67)$ & & \\
\hline$\geq 18$ & $85 / 110(77)$ & $1.7(0.4-5.9)$ & 0.417 \\
\hline \multicolumn{4}{|c|}{$\%$ total time $\mathrm{pH}<4$} \\
\hline$\geq 8 \%$ & $6 / 11(55)$ & & \\
\hline$<8 \%$ & $5 / 8(63)$ & $1.4(0.2-9.7)$ & 0.729 \\
\hline \multicolumn{4}{|c|}{ Total DeMeester score } \\
\hline$\geq 30$ & $2 / 5(40)$ & & \\
\hline$<30$ & $6 / 11(55)$ & $1.8(0.2-18.3)$ & 0.592 \\
\hline
\end{tabular}

Data are expressed $\mathrm{n} / \mathrm{n}(\%)$

$G E R D$ gastroesophageal reflux disease, GERD-HRQL gastroesophageal reflux disease health-related quality of life, GERSS gastroesophageal reflux symptom score, $O R$ odds ratio, PPIs proton-pump inhibitors, $R S I$ reflux symptom index

${ }^{\text {a }}$ Wald $p$ value

Using univariate analysis, factors identified as predicting a responsive outcome from $\mathrm{TF}$ for patients with typical GERD symptoms were the presence of esophagitis on endoscopy $\quad(\mathrm{OR}=2.6, \quad \mathrm{CI}=1.2-5.5, \quad p=0.013)$, age $\geq 50$ years $(\mathrm{OR}=2.3, \mathrm{CI}=1.1-4.9, p=0.032)$, and GERSS $>18 \quad$ on PPIs $\quad(\mathrm{OR}=2.4, \quad \mathrm{CI}=1.0-5.6$, $p=0.042$ ). Female gender and a GERD-HRQL $\geq 15$ on PPIs approached statistical significance (Table 4). Preoperative factors predictive of responsive outcome of typical GERD symptoms to TF by multivariate analysis were age $\geq 50$ years $(\mathrm{OR}=2.6, \mathrm{CI}=1.2-5.7, p=0.018)$ and the presence of esophagitis $(\mathrm{OR}=2.9, \mathrm{CI}=1.3-6.3$, $p=0.008)$, Table 3 . When both predictive factors were favorable, $84 \%(61 / 73)$ of patients achieved a responsive outcome of typical GERD symptoms. If both factors were unfavorable, $30 \%(3 / 10)$ of patients had a responsive outcome.

\section{Atypical GERD symptoms}

Of the 158 patient cohort, $78 \%(124 / 158)$ also had atypical symptoms and an RSI score on PPIs of $>13$ at presentation. In this subgroup, the median RSI score improved from 26 (14-45) to $5.5(0-41), p<0.001 .74 \% \quad(91 / 124)$ of patients were completely off PPIs, and an additional $6 \%$ (7/124) of patients were on occasional PPI therapy. $21 \%$ (26/124) of patients remained on daily PPI therapy. Median follow-up was 22 (10-43) months.

Among these patients with atypical GERD symptoms, a successful outcome (off PPIs and the total RSI score $\leq 13$ ) was observed in $60 \%(74 / 124)$ of patients. A responsive outcome (on any dose PPIs and RSI score $\leq 13$ ) was observed in $75 \%(93 / 124)$ of patients. Outcomes were considered poor in $25 \%(31 / 124)$ of patients with atypical symptoms.

The only predictor of a successful outcome of atypical GERD symptoms by univariate analysis was a total preoperative GERD-HRQL score $\geq 15$ on PPIs $(\mathrm{OR}=10.0$, $\mathrm{CI}=1.6-191.2, p=0.036)$. The presence of esophagitis approached statistical significance (Table 5).

The only predictor of a responsive outcome of atypical symptoms by univariate analysis was the presence of endoscopic esophagitis $\quad(\mathrm{OR}=3.4, \quad \mathrm{CI}=1.4-8.3$, $p=0.006$ ), Table 6 . In these patients, when esophagitis was present preoperatively, a responsive outcome was achieved in $82 \%(75 / 92)$ of patients. If preoperative esophagitis was not present, $44 \%(16 / 32)$ has a responsive outcome.

Neither duration of disease process nor duration of PPI therapy was found to be predictive of symptomatic outcomes.

\section{Complications}

Two procedures were aborted due to small esophageal tear in the distal esophagus. In both cases, 3 small hemostatic clips around the area of the esophageal tear were 
successfully used to stop a bleeding. Both patients had no clinical sequelae. All other TF procedure was completed successfully. $13 \%(21 / 156)$ of patients required hospitalization for more than $24 \mathrm{~h}$ due to nausea, anxiety, or postoperative pain. Two patients stayed in the hospital longer than 3 days for pulmonary issues, not related to the TF procedure. $42 \%(67 / 156)$ of patients reported some postoperative pain at discharge. Of these 67 patients, $4 \%$ (3/ 67 ) rated the post-operative pain as severe.

\section{Discussion}

Potent acid-suppressive therapy with PPIs heals many patients with reflux-induced esophageal injury. However, the effectiveness of medical therapy in adequately alleviating reflux symptoms in GERD patients has been more and more questioned, and many patients are dissatisfied with their QOL despite medical therapy. Although patients with a significant response to PPIs and typical GERD symptoms have a high likelihood of responding well to antireflux procedures [5], in truth most of these patients are content to stay on their medication. It is instead patients with an inadequate response to medical therapy (including those with minimal or no response to acid-suppressive therapy) who present for consideration of an antireflux procedure. These patients frequently switch from one PPI to another with little improvement in their QOL $[2,20]$ and require further work-up (multiple physician and endoscopy suite visits) [2]. This important group of patients has largely been ignored in studies of antireflux surgery, especially in the community settings. Based on our results, it appears that TF may offer those patients (who failed medical treatment and who are generally not good candidates for antireflux surgery [2]) solid symptomatic control without risks associated with traditional surgical option. In cases where TF patients experience recurrent reflux symptoms, most can achieve symptom control with cost effective over-the-counter acid-suppressive medication, as needed.

The current study evaluated predictors of successful symptomatic outcomes of one particular antireflux procedure (TF) in patients with inadequate improvement in symptoms despite PPI therapy, an abnormal QOL on PPI therapy, and objective evidence of GERD. The procedure was performed in 14 community settings using the same technology (EsophyX) by surgeons experienced in and adhering to a similar technique. At the time of study initiation, each participating investigator had performed more than $20 \mathrm{TF}$ procedures. Outcomes were measured clinically using three validated QOL questionnaires, one of which evaluates typical GERD symptoms (GERD-HRQL), one of which evaluates laryngopharyngeal (atypical) symptoms
(RSI), and one of which addresses typical and atypical symptoms (GERSS). Success was defined by a reduction in QOL scores and reduction or elimination of PPI therapy. For the purpose of this study, GERSS questionnaire was not used in defining clinical outcomes because it is not specific for typical or atypical presentation of GERD (it is composed of 2 items used to assess typical symptoms and 3 items used to evaluate atypical symptoms).

All patients in the study suffered from troublesome classic GERD symptoms. Multivariate analysis revealed that an elevated GERD-HRQL score ( $\geq 15$ on PPIs) and ages ( $\geq 50$ years) were associated with successful outcomes ( $\geq 50 \%$ reduction in GERD-HRQL scores and off PPIs). Age $\geq 50$ emerged as significant predictor of successful or responsive outcomes. As $<30 \%$ of patients in the study were in this age group, it is unclear whether this statistically significant result is of clinical importance.

Patients with typical symptoms responsive to PPI therapy can expect a good response from LF [5]. In this study we found that patients with typical symptoms that persisted at a high level despite PPI therapy ( $\geq 15$ on GERD-HRQL), and objective evidence of GERD (by endoscopy or reflux testing) could expect a good response to TF. In patients with typical symptoms, two factors associated with successful outcomes on univariate analysis (abnormal RSI score and abnormal GERSS score) did not emerge as significant predictive factors on multivariate analysis. The GERSS questionnaire covers a mixture of atypical and typical symptoms while RSI questionnaire also has a heartburn component. These questionnaires would likely have less weight in a multivariate analysis than the GERDHRQL which is limited to typical symptoms.

Unlike with successful and responsive outcomes, our study failed to determine significant predictors of poor outcomes. As reported in the previous report from the registry study [6], our population consisted of patients with more severe symptoms and symptoms that persisted despite PPI therapy. Our analysis of 10 patients who underwent revisional procedure showed that $60 \%$ of patients had high preoperative GERD-HRQL $(>30)$ and heartburn score $(>20)$ on a long-term PPI therapy. The complete cohort of the present study, similar to other TF studies [20], may represent a patient population skewed to those with more severe and medically-unresponsive symptoms. Studies of TF in patients with milder and more medically-responsive GERD symptoms may help identify the best patient population for TF [21].

Limitations of the present study include the following factors: (1) QOL assessment preoperatively was routinely performed only with the patient on acid-suppressive medication. Therefore, the degree of response to PPIs as a possible predictive factor could not be assessed; (2) Although all patients had objective evidence of GERD 
preoperatively, no single diagnostic criterion had to be met for entry. Some patients had esophagitis and no ambulatory reflux testing, others had no esophagitis but abnormal reflux testing. This limited assessment of objective criteria that might be predictive of treatment outcomes; (3) Outcomes were measured clinically with only limited objective follow-up. Although clinical outcomes are the most important, there can be confounding factors and placebo effects that limit the reliability of patients' self-reported condition. However, our study with almost 2 year followup likely negates placebo-effect. Objective testing would be helpful in deciphering these confounding factors. Currently, there are two randomized clinical trials underway with a comprehensive pre- and post-operative testing that should provide additional insights on factors associated with objective outcomes of TF.

Recognizing these limitations, the current study still provides useful information on proper patient selection for the TF procedure. Previous studies reported positive association between lower Hill grade and better outcomes after TF [11, 12]. In this study, pre-operative Hill grade was not positively associated with better outcomes. We believe that this is due to the fact that, based on these earlier studies, we excluded patients with Hill grade IV. We agree with these earlier studies that TF is not appropriate for patients with severe anatomic degradation.

In this study we defined an outcome as responsive, if patients had a good clinical response even if they continued taking PPIs (i.e., with $\geq 50 \%$ improvement in GERDHRQL score or normalization in RSI scores regardless of need for ongoing medical therapy, as long as medication use did not increase). We think this was appropriate as our patient population was inadequately controlled by PPIs, and a good therapeutic outcome can be imputed if a patient uncontrolled medically is rendered controlled medically by a therapeutic intervention. Since complete cessation of PPI therapy has been defined as "successful" by research-driven studies, we chose the term "responsive" to indicate a definable clinical outcome. We believe that more research should be devoted to evaluating patients with medicallyunresponsive GERD, and that the battle between medical and surgical therapies should instead move toward multimodality management that looks for any combination of therapies that provides the best outcomes for patients. If medically refractory symptoms are controlled by an antireflux procedure, the continued use of medication should not be construed as a failure of anti-reflux surgery.

\section{Conclusions}

This study evaluated preoperative predictors of success in 158 patients with persistent troublesome GERD symptoms despite medical therapy and who underwent an endoluminal therapy (TF). When positive predictors were present, a successful outcome $(\geq 50 \%$ reduction in GERDHRQL score and no PPI use) was seen in $67 \%$ of patients, and the presence of an elevated GERD-HRQL $(\geq 15)$ on PPIs before procedure was the best predictor of that outcome. As important if not more, given the failure of medication alone to control symptoms, $84 \%$ of patients normalized their QOL regardless of ongoing medical therapy. In this group, persistence of typical symptoms on medication (initial GERD-HRQL score $\geq 15$ on PPIs) and an objectively confirmed diagnosis of GERD (presence of esophagitis) were the best predictors of success. Though further studies will continue to define the role of $\mathrm{TF}$ in the treatment of GERD, patients with the characteristics identified herein who have persistent symptoms on PPI therapy can be offered TF with a high likelihood of having a greatly improved QOL afterward.

Acknowledgments The authors thank Robert D. Fanelli, MD, for critically reviewing the manuscript and for providing suggestions to improve the paper. The study was supported in part by a small research grant from EndoGastric Solutions for testing and data collection at follow-up.

Disclosures Reginald CW Bell and Erik Wilson have received a research grant from EndoGastric Solutions, Karim S. Trad, Reginald CW Bell, and Glenn M. Ihde have consulting agreement with EndoGastric Solutions, Brian DaCosta Gill is a paid proctor of Intuitive Surgical (da Vinci robot for antireflux surgeries) and has received honoraria from Cook BioDesign for speaking about mesh usage in paraesophageal hernia repairs, Glenn M. Ihde, Mark A Fox, Mark G Hausmann, Tanja Gunsberger, and Karim S. Trad have received honoraria from EndoGastric Solutions, and Peter Mavrelis has received honoraria from pharmaceutical companies (Santarus, Takeda, and Ironwood). William Barnes, Bart Carter, Robert Sewell, Katherine D. Freeman, Kevin Hoddinott, and David Dargis have no conflicts of interest or financial ties to disclose.

Open Access This article is distributed under the terms of the Creative Commons Attribution License which permits any use, distribution, and reproduction in any medium, provided the original author(s) and the source are credited.

\section{References}

1. Stefanidis D, Hope WW, Kohn GP, Reardon PR, Richardson WS, Fanelli RD, SAGES Guidelines Committee (2010) Guidelines for surgical treatment of gastroesophageal reflux disease. Surg Endosc 24:2647-2649

2. Katz PO, Gerson LB, Vela MF (2013) Guidelines for the diagnosis and management of gastroesophageal reflux disease. Am J Gastroenterol 108:308-328

3. Heading RC, Mönnikes H, Tholen A, Schmitt H (2011) Prediction of response to PPI therapy and factors influencing treatment outcome in patients with GORD: a prospective pragmatic trial using pantoprazole. BMC Gastroenterol. doi:10.1186/1471230X-11-52 
4. Carlson MA, Frantzides CT (2001) Complications and results of primary minimally invasive antireflux procedures: a review of 10,735 reported cases. J Am Coll Surg 193:428-439

5. Campos GM, Peters JH, DeMeester TR, Oberg S, Crookes PF, Tan S, DeMeester SR, Hagen JA, Bremner CG (1999) Multivariate analysis of factors predicting outcome after laparoscopic Nissen fundoplication. J Gastrointest Surg 3:292-300

6. Bell RCW, Mavrelis PG, Barnes WE, Dargis D, Carter BJ, Hoddinott KM, Sewell RW, Trad KS, DaCosta Gill B, Ihde GM (2012) A prospective multicenter registry of patients with chronic gastroesophageal reflux disease receiving transoral incisionless fundoplication. J Am Coll Surg 215:794-809

7. Petersen RP, Filippa L, Wassenaar EB, Martin AV, Tatum R, Oelschlager BK (2012) Comprehensive evaluation of endoscopic fundoplication using the Esophy $\mathrm{X}^{\mathrm{TM}}$ device. Surg Endosc 26:1021-1027

8. Barnes WE, Hoddinott KM, Mundy S, Williams M (2011) Transoral incisionless fundoplication offers high patient satisfaction and relief of therapy-resistant typical and atypical symptoms of GERD in community practice. Surg Innov 18:119-129

9. Trad KS, Turgeon DG, Deljkich E (2012) Long-term outcomes after transoral incisionless fundoplication in patients with GERD and LPR symptoms. Surg Endosc 26:650-660

10. Bell RCW, Freeman KD (2011) Clinical and pH-metric outcomes of transoral esophago-gastric fundoplication for the treatment of gastroesophageal reflux disease. Surg Endosc 25:1975-1984

11. Cadière GB, Buset M, Muls V, Rajan A, Rösch T, Eckardt AJ, Weerts J, Bastens B, Costamagna G, Marchese M, Louis H, Mana F, Sermon F, Gawlicka AK, Daniel MA, Devière J (2008) Antireflux transoral incisionless fundoplication using EsophyX: 12-month results of a prospective multicenter study. World J Surg 32:1676-1688

12. Testoni PA, Corsetti M, Di Pietro S, Castellaneta AG, Vailati C, Masci E, Passaretti S (2010) Effect of transoral incisionless fundoplication on symptoms, PPI use, and ph-impedance refluxes of GERD patients. World J Surg 34:750-757
13. Testoni PA, Vailati C, Testoni S, Corsetti M (2012) Transoral incisionless fundoplication (TIF 2.0) with EsophyX for gastroesophageal reflux disease: long-term results and findings affecting outcome. Surg Endosc 26:1425-1435

14. Bell RCW, Cadiere GB (2011) Transoral rotational esophagogastric fundoplication: technical, anatomical, and safety consideration. Surg Endosc 25:2387-2399

15. Velanovich V (2007) The development of the GERD-HRQL symptom severity instrument. Dis Esophagus 20:130-134

16. Velanovich V, Vallance SR, Gusz JR, Tapia FV, Harkabus MA (1996) Quality of life scale for gastroesophageal reflux disease. J Am Coll Surg 183:217-224

17. Belafsky PC, Postma GN, Koufman JA (2002) Validity and reliability of the reflux symptom index (RSI). J Voice 16:274-279

18. Allen JC, Parameswaran K, Belda J, Anvari M (2000) Reproducibility, validity, and responsiveness of a disease-specific symptom questionnaire for gastroesophageal reflux disease. Dis Esophagus 13:265-270

19. Anvari M, Allen C, Marshall J, Armstrong D, Goeree R, Ungar W, Goldsmith C (2006) A randomized controlled trial of laparoscopic Nissen fundoplication versus proton pump inhibitors for treatment of patients with chronic gastroesophageal reflux disease: one-year follow-up. Surg Innov 13:238-249

20. Hansen RA, Shaheen NJ, Schommer JC (2005) Factors influencing the shift of patients from one proton pump inhibitor to another: the effect of direct-to-consumer advertising. Clin Ther 27:1478-1487

21. Wendling MR, Melvin WS, Perry KA (2013) Impact of transoral incisionless fundoplication (TIF) on subjective and objective GERD indices: a systematic review of the published literature. Surg Endosc 27:3754-3761 